\title{
Laser-direct-write methods for fabrication of paper-based medical diagnostic sensors
}

\author{
C. L. Sones, I. N. Katis, P. J. W. He and R. W. Eason \\ Optoelectronics Research Centre, University of Southampton, Highfield, Southampton, U.K. SO17 1BJ \\ Phone: ++ 44238059 3141,Fax: ++ 44238059 3142,Email: cls@orc.soton.ac.uk
}

\begin{abstract}
We demonstrate the use of laser-based direct-write methods, namely laser-induced forward transfer and laser-induced photo-polymerization as printing and patterning tools for the fabrication of paper-based fluidic sensors that enable affordable point-of-care medical diagnostics. OCIS codes: (350.3390) Laser materials processing; (220.4610) Optical fabrication; (280.1415) Biological sensing and sensors; (160.5470) Polymers; (160.5335) Photosensitive materials
\end{abstract}

\section{Introduction}

Laser direct-write (LDW) methodologies [1] are highly flexible, non-contact and serial procedures that allow the user to create patterns either on the surface or in the volume of a material through point-by-point scanning, by moving the either the laser beam or the work-piece. Material modification and hence pattern generation can be through either addition or subtraction of the material or through modifications to its physical properties, on a scale length that can span the micron to millimetre range.

Here we demonstrate the usefulness and versatility of such LDW approaches for implementing two different processes required in the fabrication of lab-on-chip type paper-based diagnostic devices, namely, patterning of the paper devices to create the fluidic channels and reaction zones, and subsequent printing of the required biomolecules and reagents for the implementation of the biological diagnostic-detection assay. Use of paper as a platform to build affordable point-of-care diagnostic devices for use in the low-resource setting of developing countries was proposed by Whitesides et al., and since then its use for developing such diagnostic sensing devices is well-documented [2].

\section{Laser-based photo-polymerisation for patterning of paper}

An essential requirement for paper-based lab-on-chip type microfluidic devices is the need to have interconnected multiple flow channels and reaction chambers to guide and contain the fluidic reagents and biological samples on the paper-based device. These fluidic patterns should extend throughout the full thickness of the paper and several methods have been successfully explored to demonstrate the creation of fluidic designs in paper using hydrophobic materials such as SU8, PDMS, polystyrene and wax. Here we propose and demonstrate the use of a laser-based technique that relies on the principle of light-induced photo-polymerization of a commercial resist/photo-polymer for the patterning of the fluidic designs in paper.

As shown schematically (Fig. 1), a substrate (cellulose paper or a nitrocellulose membrane) is first impregnated with a photo-polymer and subsequently scanned with a laser beam at the appropriate scan speed and laser power to define the fluidic patterns. The photo-polymer in the illuminated areas undergoes polymerization and becomes insoluble in the subsequent solvent-based developing step that removes any un-polymerized material from the substrate, thereby producing the user-defined fluidic pattern in the substrate.

The lasers used for this were either a $405 \mathrm{~nm}$ continuous wave (c. w.) laser with a maximum power of $100 \mathrm{~mW}$ or a pulsed $\mathrm{Nd}: \mathrm{YVO}_{4}$ laser at $266 \mathrm{~nm}$, with a pulse duration of $10 \mathrm{~ns}$, a repetition rate of $20 \mathrm{~Hz}$ and a maximum single pulse energy of $2 \mathrm{~mJ}$. The two different photo-polymers used were DeSolite ${ }^{\circledR}$ 3471-3-14 from DSM Desotech Inc., and Substance G (SubG) from Maker Juice, USA.

By varying the direct-write parameters (such as the laser power and laser scan-speed) and hence the locally deposited laser energy density, it was possible to precisely control the extent of the local polymerization and consequently the width and depth of the polymerized pattern. For experiments with the pulsed $266 \mathrm{~nm}$ laser, the range of the translational scan speeds trialed varied from $0.05 \mathrm{~mm} / \mathrm{s}$ to $0.5 \mathrm{~mm} / \mathrm{s}$, for variations of incident average powers from $\sim 7 \mathrm{~mW}$ to $\sim 10 \mathrm{~mW}$ (corresponding to energies of $\sim 0.35 \mathrm{~mJ}$ to $\sim 0.5 \mathrm{~mJ}$ per pulse). For experiments with the c. w. $405 \mathrm{~nm}$ laser, the range of scan speeds used varied from $4 \mathrm{~mm} / \mathrm{s}$ to $10 \mathrm{~mm} / \mathrm{s}$ for corresponding variations of incident power ranging from $1 \mathrm{~mW}$ to $100 \mathrm{~mW}$.

As seen in Fig. 2, using this LDW approach it was possible to polymerize lines with widths as small as $\sim 50 \mu \mathrm{m}$. A set of such parallel lines could form the two oppositely positioned barrier-walls of a fluidic channel, and as shown in Fig. 2a and Fig. 2b, using such an arrangement, it was possible to create fluidic channels in paper (that allow the flow of the liquids) with channel widths as small as $\sim 80 \mu \mathrm{m}$. These feature dimensions (barrier-wall and channel widths) are, to our knowledge, the smallest that have been demonstrated so far, and hence should allow for further 
miniaturisation for such paper-based devices. Minimising the dimensions of the device also leads to the minimal use of reagent volumes, which would then significantly contribute towards decreasing the cost of the devices.

To further show the usefulness of our LDW procedure in diagnostic detection, we laser-patterned fluidcontaining square-well patterns in paper and then used these multiplexed fluidic tests to implement colorimetric assays for medical and environmental sensing of Bovine Serum Albumin (BSA), Glucose and Nitrite respectively (Fig 3).

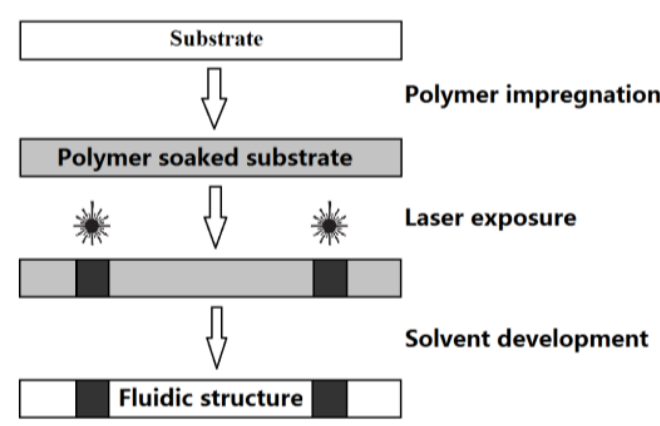

Fig. 1. Schematic of the paper patterning process.

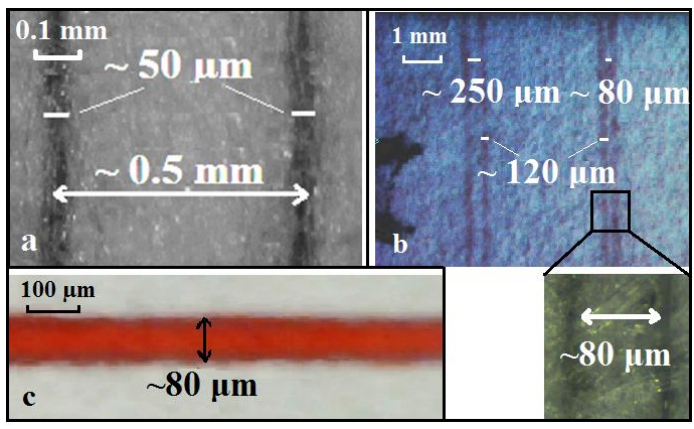

Fig. 2. a) Microscope images showing two parallel $\sim 50 \mu \mathrm{m}$ lines written via LDW, b) image showing two fluidic channels with $\sim 250 \mu \mathrm{m}$ and $\sim 80 \mu \mathrm{m}$ widths and barrier wall widths of $\sim 120 \mu \mathrm{m}$; inset shows a magnified image of the $\sim 80 \mu \mathrm{m}$ channel, c) image showing the $\sim 80 \mu \mathrm{m}$ fluidic channel guiding red ink.

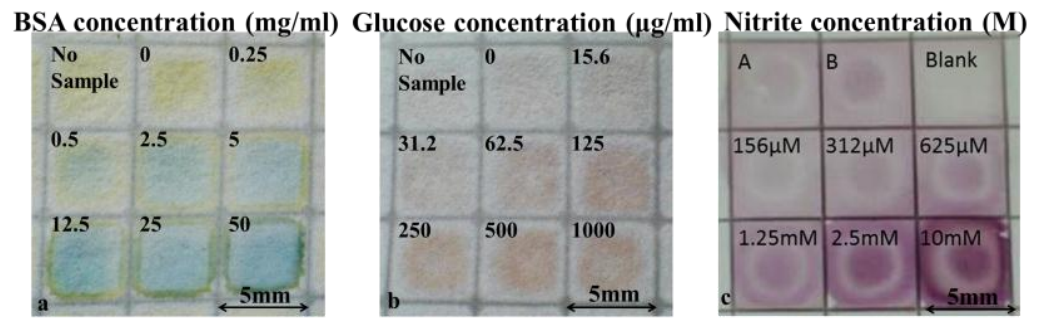

Fig. 3. Assays performed on patterned devices for the detection of a) BSA, and b) Glucose and cellulose paper, and c) Nitrite on nitrocellulose.

\section{Laser-based printing via Laser-Induced Forward Transfer (LIFT)}

To fabricate a complete diagnostic sensor on paper would also require the printing or deposition of the required chemical reagents and biological molecules at specifically desired locations of the substrate that have been prestructured with the fluidic patterns. To achieve this, we have used another LDW method called LIFT for the localised deposition on paper substrates. We now report our progress in the LIFT-printing of antibodies, both untagged and tagged/conjugated with the enzyme horseradish peroxidase (HRP). LIFT-printing of these proteins was necessary to implement an enzyme-linked immunosorbent assay (ELISA) which would in-turn establish the feasibility of our LDW method for the development of paper-based sensors.

LIFT [1], is an additive direct-write technique used for depositing materials from a thin donor film onto a receiver substrate and its schematic is shown in Fig. 4. For our experiments, the donor (a glycerol film containing antibodies) was pre-deposited onto a carrier (a fused silica substrate that is transparent to the incident laser light), and photons from the laser source $(248 \mathrm{~nm} \mathrm{KrF}$ excimer operated at $1 \mathrm{~Hz}$, with a pulse duration of $\sim 10 \mathrm{~ns}$, and delivering a maximum energy of $\sim 400 \mathrm{~mJ} /$ pulse) provided the driving force that transferred a small volume of the donor material to the receiver substrate. The threshold energy density required for LIFT-printing was $\sim 200 \mathrm{~mJ} / \mathrm{cm}^{2}$.

The viability of the untagged (target) antibodies following LIFT-transfer was validated by an indirect colorimetric ELISA. In the ELISA, the HRP-tagged detection antibody attaches specifically to the target and on addition of the corresponding chromogenic substrate, TMB, the printed pixels turn blue (Fig. 5). The colour intensities of the LIFT-printed pixels were quantified by recording the digital images with a USB camera under ambient laboratory lighting conditions, followed by analysis of the images using a customised MATLAB program. As seen in Fig. 6, the colour intensities of the LIFT-printed pixels of HRP-conjugated IgG were measured and plotted to create a calibration curve for known concentrations. An identical test was performed with the same concentrations of the HRP-conjugated antibodies via a simple pipetting procedure instead of LIFT-printing. This 
second calibration curve of the pipetted IgG was created to identify any loss of antibodies during the LIFT process (Fig. 6). Results indicated that the colour intensity of the LIFT-printed antibodies was reduced by between 2-4\% compared to the colour intensity of the pipetted antibodies. This decrease of the colour intensity can likely be attributed to the denaturing of antibodies during transfer due to the heating induced by the laser irradiation.

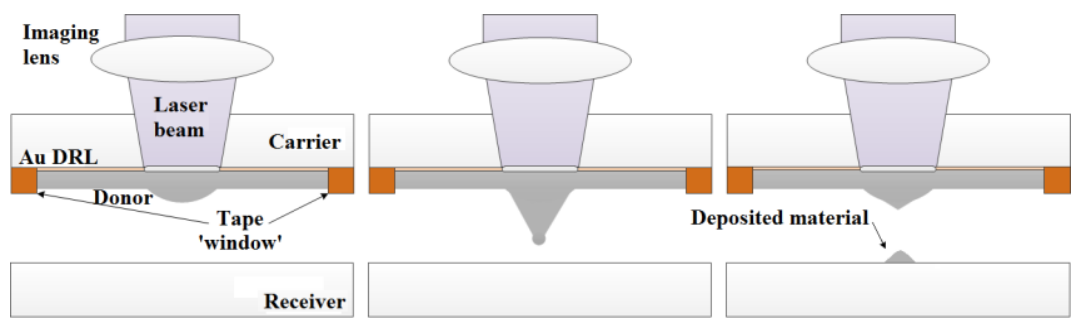

Fig. 4. Schematic of the LIFT setup for liquid donor transfer.

Fig. 6. Plot of the colour intensity of different concentrations of LIFT-printed HRP-conjugated IgG. and of pipetted HRPconjugated $\mathrm{IgG}$ along with their linear interpolation curves.

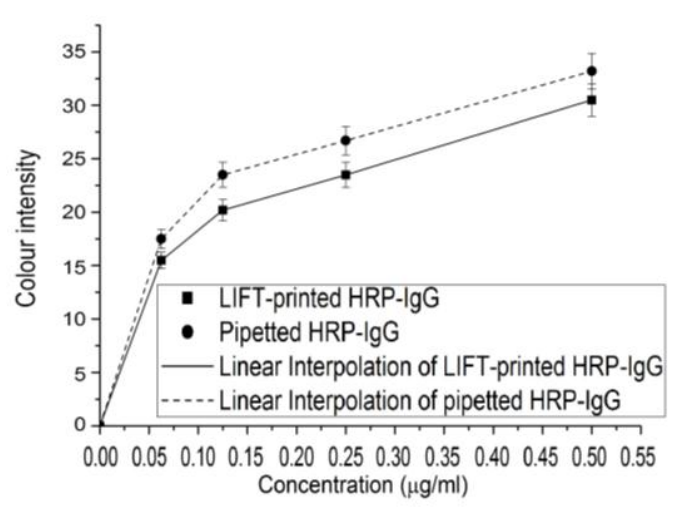

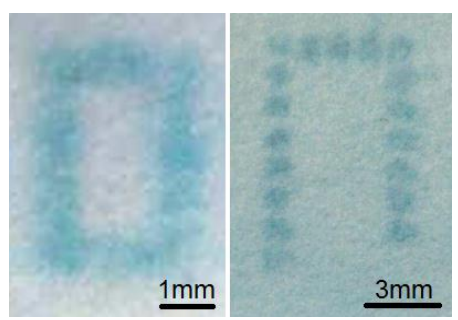

Fig. 5. Images of LIFT-printed mouse IgG after an ELISA test.

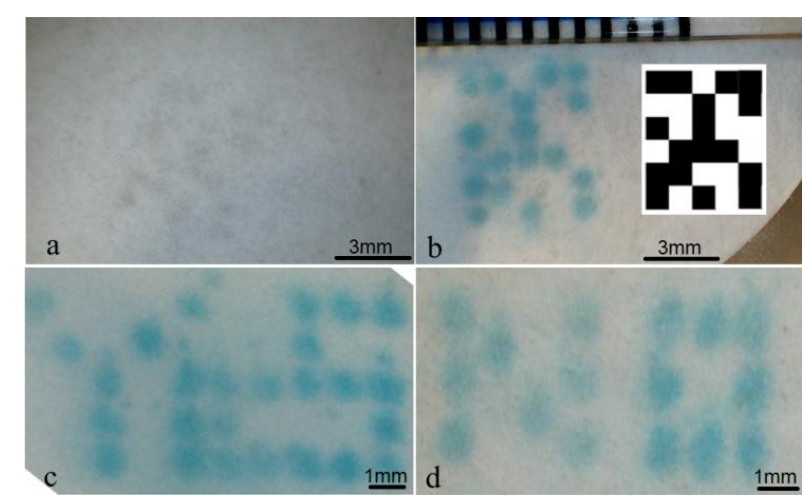

Fig. 7. LIFT-printed pixels of HRP-conjugated anti-mouse IgG in the form of a QR code, (a) before the detection with the chromogenic substrate TMB and after developing in the form of (b) a 2-d trial barcode QR code, shown as a black/white pattern in the inset, and words (c) 'yes' and (d) 'no'.

One of the important properties of LDW processing is that it allows for precise control of the size and shape of individually-printed pixels. So also, it is possible to print user-defined multiple-pixel patterns which, can be varied on an individual basis to produce patient-specific tests. Figs. 7a and 7b show the results for a simplistic LIFT-printed trial barcode, which further establishes the flexibility inherent to such a LDW procedure. The bar code patterns were revealed by addition of the chromogenic substrate TMB onto the paper after LIFT-printing of the enzyme-tagged antibody in the desired pattern. For simple tests where secrecy or patient confidentiality was not an issue, word patterns such as 'yes/no' would facilitate immediate read-out by the patient or person taking the test. Figs. $7 \mathrm{c}$ and $7 \mathrm{~d}$ show such LIFT-printed 'yes/no' patterns.

Apart from the LIFT-printing of antibodies, we are currently working on preparing diagnostic devices for the detection of BSA and glucose and also other simple colorimetric tests that can be utilized on paper. The fabrication of such a device would include the use of both the laser techniques presented here for patterning paper and printing biological molecules and reagents.

\section{Acknowledgements}

The authors acknowledge the funding received via the EPSRC Grant Nos. EP/J008052/1 and EP/K023454/1, and funding received via the Knowledge Mobilisation Fellowship for Dr. Collin Sones from the Institute for Life Sciences and the Faculty of Health Sciences of the University of Southampton.

\section{References}

[1] C. B. Arnold, P. Serra, and A. Pique, "Laser direct-write techniques for printing of complex materials," MRS Bull 32(1), 23-31 (2007).

[2] A. W. Martinez, S. T. Phillips, G. M. Whitesides, and E. Carrilho, "Diagnostics for the Developing World: Microfluidic Paper-Based Analytical Devices," Anal Chem 82(1), 3-10 (2010). 